\title{
A Initial Exploration of Coupled Transient Mechanical and Electrochemical Behaviors in Lithium Ion Batteries
}

Thomas Hodson ${ }^{1,2}$, Shripad Patil ${ }^{3}$, and Daniel A. Steingart ${ }^{3,4, z^{*}}$

${ }^{1}$ Department of Mechanical and Aerospace Engineering Princeton University, Princeton, NJ 08544, USA

${ }^{2}$ Andlinger Center for Energy and the Environment, Princeton University, Princeton, NJ 08544, USA

${ }^{3}$ Department of Earth and Environmental Engineering, Columbia University, New York, NY 10027, USA

${ }^{4}$ Department of Chemical Engineering, Columbia University, New York, NY 10027, USA

zLead Contact

*Correspondence: dan.steingart@columbia.edu

\begin{abstract}
Lithium-ion batteries represent a dominant technology in the current energy storage market. However, many aspects of these batteries are still poorly understood, and proper engineering of these aspects will lead to increased cycle life and performance parameters. In particular, the electrode/electrolyte interface is still an actively researched topic and is notoriously difficult to study. An ultrasound acoustic characterization technique is introduced to probe the electrode/electrolyte interface in operando using signal amplitude analysis. This technique gives insight into lithium ion concentration accumulation and depletion at the electrode/electrolyte interface, and these findings are consistent with Dualfoil model simulations.
\end{abstract}




\section{INTRODUCTION}

Despite the growing numbers of commercial lithium-ion batteries (LIB) throughout the world, dynamic behavior of lithium-ion systems, particularly with respect to available power at a given state of charge, remains statistically reliable though physically obscured. This is particularly true for transient chemical and mechanical behaviors at the electrode/electrolyte interface during pulsed operation of a battery. In this study we employ electrochemical-acoustic analysis to examine the real-time evolution of the electrode/electrolyte interface during operation via changes to the acoustic signal amplitude.

The electrode/electrolyte interface of lithium-ion batteries is important because it is where charge transfer occurs, allowing the electrochemical cell to operate and dictates operating conditions for the cell; as an example, the passivation layer that forms at the anode/electrolyte interface (the Solid-Electrolyte Interphase, or SEI) is known to be a key contributor to the maintenance of the coulombic efficiency of a cell, and therefore the overall cycle life of lithium-ion batteries. ${ }^{1-7}$ This interface remains poorly characterized in part due to the length scales and delicacy of operation needed to probe it. Conventional techniques have been applied to the electrode/electrolyte interface, including neutron diffraction experiments. ${ }^{8-11}$

Ultrasonic acoustic characterization tools have been used in many fields to obtain in operando measurements for decades, such as the detection of defects in large concrete structures. Additionally, acoustic emission (AE) techniques have been used in the battery community to probe degradation events in the cell. ${ }^{12-15}$ However, this has generally been limited to studying cracking events in electrode particles. Recently, ultrasonic acoustic characterization tools have been used to track changes in material properties. Sound propagation through a medium is driven by material properties, and thus changes to material properties will result in changes to how sound waves propagate. Through this technique, ultrasonic acoustic parameters have been found to track well with the State of Charge and State of Health of lithium-ion batteries ${ }^{16}$, reveal information regarding the wettability of electrodes ${ }^{17}$, and track well with overall temperature and degradation effects. ${ }^{18-21}$ It should be further noted that these measurements were taken in operando on commercial cells of different chemistries and formats, giving the community insight into evolution that typically is not present in much of the battery literature. In addition, recent ultrasonic acoustic modeling has shown the ability to detect changes in density distribution and temperature effects during cycling. ${ }^{22}$

This recent ultrasonic acoustic technique uses high frequency compressional wave pulses formed by piezoelectric transducers to record changes in material properties. Compressional pressure waves travel through both solid and liquid materials, while transverse waves only travel through solid materials (due to the non-shearing nature of liquids). The velocity of wave propagation for solid materials is defined as (equation 1): 


$$
V=\sqrt{\frac{K+(4 / 3) G}{\rho}}[1]
$$

$V$ is the velocity of wave propagation, $K$ is the bulk modulus of the medium, $G$ is the shear modulus of the medium, and $\rho$ is the density of the medium. For liquid materials, shearing forces are not present since, by definition, a fluid (of which includes liquids) deforms under shearing forces ${ }^{23}$. Thus (equation 2):

$$
V=\sqrt{\frac{K}{\rho}} \cdot[2]
$$

The velocity of an initial compressional wave through the battery cell can be calculated by taking the thickness of the cell and dividing by the time of flight (ToF) of the first arrival time of the signal. This velocity, along with a known bulk density of the battery, can be used to back out a bulk modulus for the battery cell (see Knehr). ${ }^{17}$ Note that, as a battery cell contains individual stacks of current collectors, anodes, cathode, and separators, the bulk modulus retrieved from this method will be a blend of the moduli of individual components. It should be further noted that this is not a weighted average of the moduli of individual components and is instead a Backus average of component moduli. ${ }^{24}$ Nevertheless, in a typical battery cell, the modulus of the graphite anode and developing SEI layer tend to dominate the overall blended modulus, as shown in Knehr. ${ }^{17}$. In a lithium-ion battery cell, the bulk moduli of solid components are an order of magnitude larger than liquid components. Therefore, the first arrival ToF is largely driven by solid material properties, and thus this property will only yield a partial picture of evolution at the electrode/electrolyte interface.

There are, however, other ultrasonic acoustic parameters that can provide direct insight into the electrode/electrolyte interface. As an acoustic wave travels through media and comes to an interface, a portion of the wave will be transmitted and a portion will be reflected. The proportion of wave reflectance versus transmittance is governed by acoustic impedance mismatching. ${ }^{23}$ Every material has an acoustic impedance value, which can be thought of as the resistance of a material to the propagation of an acoustic wave. Acoustic impedance $(Z)$ is defined as:

$$
Z=\rho V[3]
$$

Using equations 1 and 2, we can see the relationship between acoustic impedance, density, and moduli:

$$
\begin{gathered}
Z=\sqrt{(K+(4 / 3) G) \rho} \text { and [4] } \\
Z=\sqrt{K \rho} .[5]
\end{gathered}
$$

In order to analyze the impedance mismatch between two materials at an interface, one can compare the acoustic impedances of each material. It is assumed that the incoming wave will be perpendicular to all interfaces, as this will simplify calculations. The reflectance and transmittance of the wave can thus be calculated $\mathrm{as}^{25}$ : 


$$
\begin{gathered}
\text { Reflectance }=\left(\frac{Z_{1}-Z_{2}}{Z_{1}+Z_{2}}\right)^{2} \text { and [6] } \\
\text { Transmittance }=\frac{4 Z_{1} Z_{2}}{Z_{1}+Z_{2}} .[7]
\end{gathered}
$$

It should be noted that $Z_{1}$ is the larger of the two acoustic impedances, and Reflectance + Transmittance sums to a value of one. At every interface the wave propagates through, the amount of the wave that transmits through the interface is a function of material properties. The wave propagation is thus dependent on these interfaces and serves as a great indicator for changes that occur at the interface. In practice, however, measuring the acoustic impedance as well as rates of transmission and reflection of the signal are difficult to achieve and instead the signal amplitude is measured. Signal amplitude is a measure of voltage excitation at the piezoelectric element of the receiving transducer; as the pressure of an incoming compression wave peaks, the voltage at the piezoelectric element increases and similarly voltage decreases during a pressure trough. These compression waves are in a sense a convolution of overlapping transmitted waves, and so the signal amplitude is proportional to the degree of overall transmission in the entire cell:

$$
\text { "Signal Amplitude" } \propto \text { Transmittance }{ }_{\text {overall }} \text { [8] }
$$

Hence the acoustic amplitude of the wave at the receiving end, a proxy for wave transmittance, should change as the material properties at these interfaces change. Given the porous nature of the electrodes and separator materials, liquid electrolyte is found throughout the cell and can therefore contribute to changes in acoustic signal amplitude. Changes in acoustic signal amplitude that can be traced to changes occurring at the electrode/electrolyte interface, as well as changes occurring to the solid and liquid components themselves. These changes are further compared to simulations of lithium-ion batteries in the Dualfoil battery simulation model which implements the Doyle-Fuller-Newman phase superposition model. ${ }^{26-28}$ This article serves to understand how the ultrasound technique can be applied to analysis of the electrolyte, its connections to models such as the Dualfoil simulation, and how such trends evolve over longer cycling.

\section{EXPERIMENTAL PROCEDURES}

Electrochemical and ultrasonic acoustic tests were conducted simultaneously on commercial lithium ion pouch cells in a temperature controlled environment. Unless otherwise stated, the cycling temperature was kept to $20^{\circ} \mathrm{C}+/-0.5^{\circ} \mathrm{C}$. The lithium ion pouch cells (from manufacturer BatterySpace where not noted) contained a graphite negative electrode and a lithium cobalt oxide positive electrode (data from lithium pouch cells containing a graphite negative electrode and a lithium iron phosphate positive electrode are shown in the Supplementary Materials). The fresh lithium ion pouch cells were initially fully charged and then fully discharged at a $\mathrm{C} / 2$ rate at room temperature before being placed in the temperature controlled environment and allowed to equilibrate. Cells were cycled either using a Neware 
BTS3000 battery cycler or a Keithley 2400 SourceMeter potentiostat (both using a four-wire set up). Unless otherwise stated, the cycling protocol for the cells was a $\mathrm{C} / 2$ constant current rate determined from nominal capacity for both charging and discharging. For long term cycling, a $\mathrm{C} / 2$ constant current, constant voltage cycling protocol with a $\mathrm{C} / 10$ rate current cutoff was established for charging the cell in order to fully intercalate the graphite anode during each successive charging cycle.

A custom, 3D printed holder was used to place the transducers flush against the surface of the battery pouch cell. This setup is shown in Fig. S1, along with connections to the battery cycler and ultrasound equipment. An Olympus Epoch 600 ultrasonic pulser-receiver and CompactPulser ultrasonic pulser-receiver were used for collection of ultrasonic acoustic data. Ultrasonic pulses were transmitted and received using SIUI $2.5 \mathrm{MHz}$ piezoelectric transducers, with ultrasonic couplant gel applied to the surface of the battery and the surface of the transducer. Cycling and ultrasonic acoustic measurements were taken every 10 seconds. Multiple cells were tested at each condition to guarantee reproducibility of results.

\section{RESULTS}

In our experiments, the ultrasonic acoustic signal amplitude was analyzed over an initial charge/discharge cycle for a fresh commercial cell. An example of this data is shown in Fig. 1. The ultrasonic acoustic signal amplitude was normalized such that an initial amplitude value at rest in a fully discharged state was taken to be a value of zero, while the amplitude value at rest in a fully charged state was taken to be a value of one. This trend is shown in the normalized amplitude panel of Fig. 1. Our data are normalized in order to better compare subsequent run conditions: slight variations in acoustic transducer placement or amount of ultrasonic couplant can result in changes to raw ultrasonic acoustic signal amplitude values. As a way to mitigate these effects on the trends, the data are normalized.

\section{[Insert Figure 1]}

Additionally, in the panel below normalized amplitude in Fig. 1, a Time of Flight (ToF) Shift trend is displayed. The ToF of an acoustic signal is the time $t_{0}+d t$ after an initial wave pulse at time $t_{0}$. For instance, a ToF value of 3 microseconds translates to a measurement taken 3 microseconds after an initial pulse wave was applied at the input. It has been shown that, while the overall acoustic signal waveform does not change much during cycling, the waveform moves back and forth in temporal ToF space during cycling. ${ }^{16}$ An in-house convolution algorithm was used to quantify this movement in temporal ToF space. For a more thorough discussion on ToF Shift, see Davies or Knehr. ${ }^{16,17}$ Below the normalized amplitude and ToF Shift panels are electrochemical data, measured voltage and C-Rate (where C-Rate is defined as the applied current divided by the nameplate capacity).

[Insert Figure 2] 
An interesting trend is noted in the normalized amplitude that does not appear in the ToF Shift or the electrochemical data. When the cell begins charging, noted with a green colored background, the signal amplitude dips lower before eventually monotonically increasing. At the end of charging, the signal amplitude spikes up when current is no longer applied before quickly plateauing to a stable value. A reverse trend is seen upon discharging the cell, noted by a red colored background, where the signal amplitude spikes up, decreases monotonically, and eventually jumps back down after current is no longer applied. This trend is highlighted in Fig. 2, where the initial charge and discharge amplitude 'blips' are provided in zoomed inset figures. Note the presence of a small spike up in amplitude towards the end of discharge that is also present in the ToF Shift; we believe this is related to a possible phase change in one or more of the electrode materials, resulting in drastic changes in the materials properties of the cell. This trend is outside the scope of this paper, as we will focus solely on the bulk shifting of the normalized amplitude curve during charging and discharging.

\section{[Insert Figure 3]}

This bulk shifting of normalized amplitude is independent of the initial State of Charge (SoC) upon charging or discharging, shown in Fig. 3. It is noted that charging or discharging at various SoCs has little effect on both the magnitude and duration of the shift, with the shift in amplitude occurring roughly over the course of one to two minutes. These trends are seen when the transducers are reversed in direction (see Fig. S2) as well as with different ultrasonic acoustic pulser equipment and different manufacturers of the same cell chemistry (see Fig. S3). Additionally, these trends are seen with a different cathode chemistry (lithium iron phosphate, or LFP, seen in Fig. S4). Thus, it appears these trends are invariant to external parameters and are therefore intrinsic to the battery materials themselves, particularly those components shared between LCO and LFP cell chemistries (i.e., the graphite anode and carbonate-based electrolyte). This is in agreement with previous findings that the graphite anode dominates changes to ultrasonic parameters in graphite/transition metal oxide lithium-ion systems. ${ }^{16}$

\section{DISCUSSION}

The above data yields a few key insights into what is driving this bulk shift in normalized amplitude. First, this trend is seen exclusively in the normalized amplitude of the acoustic signal and not the ToF Shift (see Fig. 1). As mentioned in the Introduction section, as a result of non-shearing effects of the liquid electrolyte, the majority of the trends seen in a ToF Shift analysis are due to changes in solid parameters. Thus, given that the normalized amplitude incorporates changes to both solid and liquid phases, any changes occurring in the liquid electrolyte will result in changes to the normalized amplitude. Second, the bulk shift in amplitude reverts once current is no longer applied, occurring on the same order of magnitude in time as the initial shift up or down in amplitude. Given that changes in bulk material properties happen on the same order of the time that it takes for species to diffuse in the system, it is assumed that this process is a reversible, physical change occurring in the system. 
The reversible nature of this bulk shift is further noted in Fig. 3, wherein the invariance of the bulk shift with regards to $\mathrm{SoC}$ is seen. Given that solid material properties as well as solid-state diffusion in the electrode particles vary throughout the SoC range ${ }^{29-32}$, it can be deduced that this bulk shift in amplitude is likely related to a physical change occurring at the interface or with the electrolyte (or both), as changes to the electrolyte are not dependent on SoC. Furthermore, it is interesting to note that LFP commercial cells show similar trends in normalized amplitude, including the bulk shift (see Fig. S4). While the cathode materials of the commercial LCO and LFP cells are different, the anode materials as well as the electrolyte solution are similar. This suggests that material properties at the anode and the anode/electrolyte interface are responsible for a majority of the trends seen in the ultrasonic acoustic data, a finding that is consistent with previous results. ${ }^{16}$

With these insights, we look to battery simulations and models to clarify the ultrasonic acoustic trends seen experimentally. The heavy dependence of the ultrasonic acoustic technique on materials properties suggests a concentrated solution model with porous electrode theory can give insight into the trends, as these models yield realistic depictions of material states during battery operation. The Dualfoil model developed by Newman has remained one of the dominant models in the LiB community for its speed as well as empirically-derived relations developed from over a decade's worth of research. ${ }^{26-28,33,34}$ The Dualfoil model outputs lithium ion concentration in the liquid electrolyte phase as well as intercalated lithium stoichiometry in the solid phase, among other parameters; again, as the anode material properties dominate ultrasonic acoustic trends, our focus will be the average lithium ion concentrations in the liquid electrolyte around anode particles as well as the averaged intercalated lithium stoichiometry in anode particles.

\section{[Insert Figure 4]}

An example of this Dualfoil output data is seen in Fig. 4 for a single charge and discharge cycle, the same protocol seen in Fig 2. Dualfoil model parameters can be found in the Supplementary Methods section. The average lithium ion concentrations in the liquid electrolyte around anode particles and the averaged intercalated lithium stoichiometry in anode particles are shown in the left panels, while a normalized weighted sum of both is seen in the panel on the right. While the averaged intercalated lithium stoichiometry in anode particles is not itself a percent change, it can be thought of as a proxy for the change in solid-state concentration of lithium in the anode particles. Thus, one can sum these values as each is inherently non-dimensional. Note that this is not a standard technique and is done in order to analyze changes in Dualfoil output data for the solid and liquid components of the system. The summation is of the following form:

$$
\text { "Normalized Amplitude" }=\alpha\left(L i_{e, a}^{+} / L i_{e, a, 0}^{+}\right)+\beta\left(L i_{s, a}\right) \text {. [9] }
$$


Here, $\mathrm{Li}_{e, a}^{+}$is the instantaneous concentration of lithium-ions around the anode, $\mathrm{Li}_{e, a, 0}^{+}$is the concentration of lithium-ions around the anode at steady-state, and $L i_{s, a}$ is the concentration of lithium intercalated into the anode particles. $\alpha$ and $\beta$ in this instance are weighting constants; we can combine these two into a single weighted constant by dividing out the $\beta$ as follows:

$$
\text { "Normalized Amplitude" }=\xi\left(L i_{e, a}^{+} / L i_{e, a, 0}^{+}\right)+\left(L i_{s, a}\right) .[10]
$$

Thus, $\xi$ can be thought of as a normalized weighting constant that compares the effects of changes in lithium ion concentration in the electrolyte to solid-state lithium concentration in the solid anode particles (for our base case, $\xi=2.82$ ). A value of $\xi>1$ implies that the normalized amplitude has a stronger dependence on changes in lithium ion concentration in the electrolyte than solid-state lithium concentration in the solid anode particles. The data is then normalized under the same normalization process done for the experimental data (i.e., an initial amplitude value at rest in a fully discharged state was taken to be a value of zero, while the amplitude value at rest in a fully charged state was taken to be a value of one).

The result of this process is the "Normalized Amplitude" data seen in the right panel on Fig. 4. Note the introduction of a bulk shifting of this amplitude data due to the depletion of lithium ion concentration in the electrolyte around the anode particles during charging, and an accumulation of lithium ion concentration in the electrolyte around the anode particles during discharging. The concentration gradient that develops in the electrolyte around the anode can be attributed to a mismatch between diffusion of lithium ions in the electrolyte and the intercalation/deintercalation of lithium ions in to and out of the surface of the anode particles. . $35-37$ That is, upon the application of a charging current to the system, lithium ions in the electrolyte surrounding the anode particles are quickly intercalated into sites available at the electrode surface. This causes a precipitous drop in the average concentration of lithium ions in the electrolyte, a drop that is then arrested by diffusion of lithium ions towards the electrode particles. An equilibrium is met between ions intercalating into the electrode and ions diffusing toward the electrode to fill in that gap. When current is no longer applied, lithium ions continue to diffuse into the depleted region until the concentration gradient equilibrates. A similar trend occurs upon application of a discharging current to the system, wherein intercalated lithium at the surface of the anode particles deintercalates and enters the electrolyte.

\section{[Insert Figure 5]}

As shown in Fig. 5, this model of normalized amplitude matches experimental data quite well. A value of $\xi=2.82$ was chosen to best fit the initial decrease and increase in amplitude during charging and discharging, respectively. Note that the experimental data becomes slightly shifted with respect to the Dualfoil simulation data after the initial charge due to a slight underand over-performance of the cell (i.e., C/2 rate charge and discharge take slightly less than 2 hours to reach the voltage cutoff, while $\mathrm{C} / 10$ rate charge and discharge take slightly more than 10 hours). Additionally, we reiterate that the shift up in amplitude towards the end of discharge is 
related to a different phenomena than the bulk shifting of the amplitude curve, and is therefore outside of the scope of this paper. In the right panel of Fig. 5, we see that our Dualfoil model matches well with a $\mathrm{C} / 2$ rate at $20^{\circ} \mathrm{C}$. At slower cycling rates, the bulk shifting of the amplitude curve tends to disappear. This trend is captured in our Dualfoil simulation and is shown in the left panel of Fig. 5 for a $\mathrm{C} / 10$ rate (note the slight over-performance of the cell in this case).

\section{[Insert Figure 6]}

At higher cycling rates, the bulk shift in amplitude becomes more pronounced. For the same value of $\xi(\xi=2.82)$, higher rate performance is also predicted. In Fig. 6 , subsequent $\mathrm{C} / 2$, $1 \mathrm{C}$, and $2 \mathrm{C}$ rate cycles are shown for experimental data in the left panels and for Dualfoil model data in the right panels. For the $2 \mathrm{C}$ cycle, note that the amplitude does not change in value much during charging. As this particular LiB is only rated for a maximum of $1 \mathrm{C}$ rate charge, this lack of change in amplitude might be explained by an increase in side reactions and hence little intercalation of the graphite anode particles. This would explain the discrepancies between resting voltage for $2 \mathrm{C}$ discharge in the experimental and simulation cases, as well as discrepancies in the normalized amplitude and subsequent differences between peaks in amplitude during the $2 \mathrm{C}$ discharge.

\section{Connections between Ultrasonic Acoustic Amplitude and Lithium Concentrations}

It has been shown in previous literature that changes in bulk material properties correspond to changes in ultrasonic acoustic propagation. ${ }^{16,17,23,38-45}$ In particular, State of Charge ( $\mathrm{SoC})$ and State of Health $(\mathrm{SoH})$ changes correspond to almost linear changes in Time of Flight (ToF) Shift and acoustic signal amplitude. ${ }^{16}$ This can be explained by almost linear changes to material properties in the graphite anode, particularly the bulk modulus of lithiated graphite. ${ }^{31,46-49}$ Hence, the stoichiometry number of $x$ in $\mathrm{Li}_{\mathrm{x}} \mathrm{C}_{6}$ that Dualfoil outputs (a proxy for the SoC of the cell) is a sufficient measure for changes to the bulk modulus of the graphite anode. Much of the linear trends seen during charging and discharging can be described by changes to the stoichiometry number, $x$, alone; however, the stoichiometry number does not account for the bulk shifting of the amplitude curve in the initial moments after current is applied. The stoichiometry number, $x$, does show relaxation when current is turned off, and this is seen slightly in the lower left panel of Fig. 4. However, this relaxation is not present at the beginning of charging or discharging, and furthermore the relaxation trend of the stoichiometry number is opposite the trends seen at the end of charging and discharging.

The concentration of lithium ions in the electrolyte around the electrode particles also changes rapidly when current is applied. In the upper left panel of Fig. 4, it is shown that the lithium ion concentration in the electrolyte around the anode undergoes a bulk shift up and down during discharging and charging, respectively, effectively creating accumulation and depletion regions of lithium ion concentration. Future experiments involving a symmetric cell setup would be able to discern if these trends are localized to one electrode interface or the degree that each 
interface contributes. These accumulation and depletion regions disappear as quickly as they are formed once the cell is placed at rest. These regions effectively modulate the overall propagation of an ultrasonic acoustic wave by changing what happens to the wave at the electrode/electrolyte interface. Referring back to Eqns. [6] and [7], it is shown that a larger acoustic impedance mismatch results in greater reflection and less transmission of the acoustic wave through the interface. Given that bulk moduli and densities of the solid components are larger than those of the liquid components, the acoustic impedance value of the solid electrode particles are greater than that of the liquid electrolyte. A decrease in lithium ion concentration in the liquid electrolyte would result in a localized decrease in density, which in turn would result in a lower acoustic impedance due to lowered densities. In turn, this would result in a larger impedance mismatch between the solid and liquid components of the system, and this would ultimately attenuate the wave more that results in a lower acoustic amplitude. On the other side, an increase in lithium ion concentration in the liquid electrolyte that results in a higher acoustic impedance would ultimately attenuate the wave less and result in a higher acoustic amplitude. These trends would simply explain the bulk shifting phenomena seen in the experimental normalized amplitude data.

\section{[Insert Figure 7]}

The effects of electrolyte on the bulk shifting of the amplitude curve are further seen experimentally after long-term cycling. Fig. 7 shows the changes in normalized amplitude for a single charge and discharge cycle over the first 96 cycles, and from this we can note changes that occur both to the amplitude curve overall as well as particular changes to how the curve shifts. In the left panel of Fig. 7, it is noted that after the first 6 cycles, a break-in period occurs wherein the cell amplitude increases at every point in the charge/discharge cycle. As stated in previous work, these cells undergo a period of electrolyte penetration during this break-in period. ${ }^{17}$ Thus, with more electrolyte surrounding electrode particles, there is less of an impedance mismatch at each interface and hence an increase in signal amplitude at every point. In the middle panel of Fig. 7 , it is noted that after this initial increase in signal amplitude, the amplitude slowly drifts down during cycling (however, the amplitude values are roughly the same at bottom of charge resting). This implies that the cell is fully discharged once it hits its lower voltage cutoff; however, after cycling, the cell fails to reach a fully charged state once hitting the upper voltage cutoff limit. This could be the result of gas formation occurring at the interface, and the gas present results in a larger impedance mismatch that results in more of the acoustic signal being reflected.

While there are bulk changes to the amplitude during cycling, the bulk shifting of the amplitude curve upon charging and discharging remains roughly consistent through these cycles. The shift occurs rapidly over the course of one to two minutes and afterwards is subsumed by the linear trend of intercalation in the graphite anode. However, around cycle 80, it is noted that this cell began to undergo characteristic gassing. This was noted by a steady drop both in cell capacity and signal amplitude at every point, including during rest. From the right panel of Fig. 7, we see that the amplitude for cycle 96 has fallen well below cycle 56, and it is 
noted that the amplitude at bottom of charge resting is below its consistent value of roughly 0.1 and below its initial value of 0.0 . The gassing event was later confirmed via visual inspection of the cell, shown in Fig. 8.

\section{[Insert Figure 8]}

Electrolyte reduction is generally the source of gas formation in lithium-ion batteries. ${ }^{7,9,50-53}$ Gassing has occurred in the cycled cell, as evidenced by the puffed nature of the cell (see Fig. 8). Therefore, the electrolyte concentrations and availability at cycle 96 is different than at cycles before the gassing event began. These changes in electrolyte properties manifest themselves as changes in the bulk shifting of the amplitude curve during cycling. Referring again to the right panel of Fig. 7, it is noted that, while the bulk shifting still occurs, the amplitude plateaus to a steady value before continuing on its linear trend. This extends the shift from happening on the order of one to two minutes to upwards of half an hour. It is noted that this drift happens fairly abruptly and is not seen in early cycles, further suggesting that this is driven by gas formation from electrolyte reduction. Given this, it is fair to assume that changes in the electrolyte affect the bulk shifting of the amplitude curve, giving further credence to the role that lithium ion concentration has on ultrasonic acoustic amplitude.

\section{Temperature effects on bulk shifting of Ultrasonic Acoustic Amplitude}

Given the temperature dependence of material properties, particularly the liquid electrolyte, the effects of temperature on the bulk shifting of the Ultrasonic Acoustic Amplitude were studied. Experimental data for a $1 \mathrm{C}$ rate charge and discharge are shown in Figs. 9a and $9 \mathrm{~b}$, while corresponding Dualfoil simulations of the experimental data are shown in Figs. 9c and 9d. Ambient temperatures are noted in the figure legends. Battery cells were charged and discharged at room temperature before being transferred to a temperature controlled environment and held for at least 48 hours. The drawbacks of the Dualfoil simulation model when incorporating temperature changes become apparent fairly quickly, as shown by under-prediction of the cell over-potential in the Dualfoil simulation when compared to experimental data, particularly at colder temperatures (see Potential panels for Figs. 9a-d). That said, we can see in the normalized amplitude panels of Figs. 9a-d that the bulk shifting of amplitude is still captured.

\section{[Insert Figure 9]}

Referring back to equation [9], note the presence of the $\xi$ constant. While $\xi$ has been shown to be roughly invariant to current rate for a given temperature, $\xi$ is certainly a function itself of temperature as electrolyte and electrode material properties vary differently as a function of temperature. Thus, $\xi$ values were adjusted in Figs. $9 \mathrm{c}$ and $9 \mathrm{~d}$ in order to match the experimental data. These $\xi$ values as a function of temperature are shown in Fig. 10; note that 
at elevated temperatures, $\xi$ values for charge and discharge shifting diverge and must be evaluated separately.

\section{[Insert Figure 10]}

There are a few important findings that can be inferred from analysis of $\xi$ temperature dependence. First, under normal operating temperatures for a $\mathrm{LiB}, \xi$ is greater than one, implying that changes to the electrolyte have a greater effect on the bulk shifting of amplitude than changes to the electrode. This is a verified since electrolyte conductivity varies more than solid-state diffusion in the electrode materials under this temperature regime. ${ }^{54,55}$ Second, the deviations in $\xi$ values on charging and discharging at elevated temperatures may give insight into electrolyte performance for charging versus discharging. For instance, it is known that the cell heats up more upon discharge, and this extra heating combined with hindered heat transfer at elevated temperatures could partially explain the divergence in $\xi$ values. Other factors, such as differences in the kinetics of intercalation versus deintercalation of the electrode materials or effects from parasitic reactions, could also play a role in $\xi$ temperature dependence.

The connection between $\xi$ and the bulk shifting in amplitude can be used by researchers to evaluate the effectiveness of electrolyte additives and general performance of the electrolyte in lithium-ion batteries. It has been frequently shown in the literature that concentration gradients in electrode particles can cause stress formation and fracture $21,35,56-65$; hence, concentration gradients forming in the electrolyte at the electrode/electrolyte interface will certainly have undesired consequences for the cycle life and performance of the LiB. Further analysis of this $\xi$ factor would give insight into these undesired consequences. The value of $\xi$ at room temperature, 2.82 , itself does not have physical significance, but rather can be used as a comparison tool to compare the interactions between various cell components. For instance, for a given set of electrode materials and operating conditions, a higher $\xi$ factor indicates a larger buildup of ions during cell operation and thus may indicate a less stable electrolyte formulation.

\section{CONCLUSION}

An ultrasonic acoustic technique for analyzing interfacial evolution at the electrode/electrolyte interface has been shown. The technique utilizes variations in acoustic impedance mismatch at the electrode/electrolyte interface to give insight into changes in concentrations at the interface. Specifically, relatively fast changes that result in an accumulation or depletion of lithium ions in the electrolyte near anode particles contrast the slower process of mostly linear solid moduli variations as a result of lithium intercalation. These accumulation and depletion regions result in a bulk shifting of ultrasonic acoustic signal amplitude as a result of a decrease and increase in acoustic impedance, respectively. The bulk shifting of amplitude is shown to be independent of transducer orientation, State of Charge, cell manufacturer, and cathode cell chemistry while varying based on current rate and temperature. A Dualfoil model is shown to explain these trends well, matching current rate variations. The 
Dualfoil model is based on a weighted summation lithium concentrations in the solid electrode phase and the ionic electrolyte phase, with a weighting factor $\xi$. An example of electrolyte degradation is shown to result in changes to the bulk shifting of amplitude, further resolving the source of the experimental trends. Additionally, $\xi$ is shown to vary heavily with temperature, and thus further analysis of this weighting parameter may yield further insights into interfacial evolution. 


\section{ACKNOWLEDGEMENTS}

This work was supported by grants from DOE APRA-E DE-AR000621. Thomas Hodson was supported through ExxonMobil while at Princeton where he learned the acoustic time of flight method. The authors thank Greg Davies and Wesley Chang for useful discussions.

\section{AUTHOR CONTRIBUTIONS}

T.H. and D.A.S. developed the idea and drew conclusions from data. T.H. and S.P. provided analysis of the ultrasonic signals. T.H. and S.P. conducted experiments. T.H. wrote the manuscript.

\section{DECLARATION OF INTERESTS}

Daniel A. Steingart is a founder of Feasible, Inc which employs the ultrasonic technique (patent US10132781B2) used herein. Feasible did not fund the work nor participate in its execution.

\section{SUPPLEMENTARY METHODS}

\section{Dualfoil Model Parameters}

For our simulations, much of the default Dualfoil parameters were left unchanged so as to give a baseline approximation of a commercial LiB. The variable temperature flag, 'lht', was set to 0 and the heat transfer coefficient, ' $h t c$ ', was set 0.368 to enable temperature fluctuations. Unless otherwise specified, the ambient temperature, 'Tam', and initial cell temperature, ' $\mathrm{T}$ ', were set to $20^{\circ} \mathrm{C}$. The thickness of the anode, ' $\mathrm{h} 1$ ', was set to $96 \mu \mathrm{m}$ while the thickness of the cathode, ' $h 3^{\prime}$, was set to $60 \mu \mathrm{m}$ in order to better model the cathode limited nature of modern commercial lithium-ion batteries. The anode material, 'nneg', was set to 6 (Lonza KS6 graphite (Bellcore)) as opposed to the default value of 3 (MCMB 2528 graphite (Bellcore)) due to the latter's over-fitting of lithium staging in graphite. For a visual description of how this changed our simulation, see Fig. S5. 


\section{REFERENCES}

1. K. Xu, Chem. Rev., 114, 11503-11618 (2014) http://dx.doi.org/10.1021/cr500003w.

2. O. C. Harris, Y. Lin, Y. Qi, K. Leung, and M. H. Tang, J. Electrochem. Soc., 167 (2020) http://jes.ecsdl.org/content/167/1/013502.abstract.

3. O. C. Harris, K. Leung, and M. H. Tang, J. Electrochem. Soc., 167 (2020) http://jes.ecsdl.org/content/167/1/013503.abstract.

4. V. A. Agubra and J. W. Fergus, J. Power Sources, 268, 153-162 (2014) http://www.sciencedirect.com/science/article/pii/S0378775314008775.

5. P. M. Attia, S. Das, S. J. Harris, and M. Z. Bazant, Journal of The (2019) http://jes.ecsdl.org/content/166/4/E97.short.

6. T. R. Ashwin, A. Barai, K. Uddin, L. Somerville, A. McGordon, J. Marco, J. Power Sources, 385, 141-147 (2018) http://www.sciencedirect.com/science/article/pii/S0378775318302350.

7. P. Verma, P. Maire, and P. Novák, Electrochim. Acta, 55, 6332-6341 (2010) http://www.sciencedirect.com/science/article/pii/S0013468610007747.

8. H. Chang, A. J. Ilott, N. M. Trease, M. Mohammadi, A. Jerschow, and C. P. Grey, J. Am. Chem. Soc., 137, 15209-15216 (2015) http://dx.doi.org/10.1021/jacs.5b09385.

9. B. Michalak, H. Sommer, D. Mannes, A. Kaestner, T. Brezesinski, and J. Janek, Sci. Rep., 5, 15627 (2015) http://dx.doi.org/10.1038/srep15627.

10. V. Zinth, C. von Lüders, M. Hofmann, Jo. Hattendorff, I. Buchberger, S. Erhard, J. Rebelo-Kornmeier, A. Jossen, R. Gilles, J. Power Sources, 271, 152-159 (2014) http://www.sciencedirect.com/science/article/pii/S0378775314012233.

11. Z. Nie, P. McCormack, H. Z. Bilheux, J. C.Bilheux, J. P. Robinson, J. Nanda, G. M. Koenig Jr., J. Power Sources, 419, 127-136 (2019) http://www.sciencedirect.com/science/article/pii/S0378775319302009.

12. K. Rhodes, M. Kirkham, R. Meisner, C. M. Parish, N. Dudney, and C. Daniel, Rev. Sci. Instrum., 82, 075107 (2011) http://dx.doi.org/10.1063/1.3607961.

13. K. Rhodes, N. Dudney, E. Lara-Curzio, and C. Daniel, J. Electrochem. Soc., 157, A1354-A1360 (2010) http://jes.ecsdl.org/content/157/12/A1354.abstract.

14. C. Villevieille, M. Boinet, and L. Monconduit, Electrochem. commun., 12, 1336-1339 (2010) http://www.sciencedirect.com/science/article/pii/S1388248110003127.

15. J. Bernard, US Patent (2015)

https://patentimages.storage.googleapis.com/3e/1a/e8/f261c6669bd7d5/US8984944.pdf.

16. G. Davies, K. W. Knehr, B. Van Tassell, T. Hodson, S. Biswas, A. G. Hsieh, and D. A. 
Steingart, J. Electrochem. Soc., 164, A2746-A2755 (2017)

http://jes.ecsdl.org/content/164/12/A2746.abstract.

17. K. W. Knehr, T. Hodson, C. Bommier, G. Davies, A. Kim, and D. A. Steingart, Joule, 2, 1146-1159 (2018) http://www.sciencedirect.com/science/article/pii/S2542435118301314.

18. C. Bommier, W. Chang, J. Li, and S. Biswas, Journal of The (2020)

https://iopscience.iop.org/article/10.1149/1945-7111/ab68d6/meta.

19. W. Chang, C. Bommier, T. Fair, and J. Yeung, Journal of The (2020)

https://iopscience.iop.org/article/10.1149/1945-7111/ab6c56/meta.

20. C. Bommier, W. Chang, Y. Lu, J. Yeung, G. Davies, R. Mohr, M. Williams, and D. Steingart, Cell Reports Physical Science, 1, 100035 (2020)

http://www.sciencedirect.com/science/article/pii/S2666386420300254.

21. J. H. Park, A. Raj, A. Kim, and G. Davies, Microscopy (2018)

https://www.cambridge.org/core/journals/microscopy-and-microanalysis/article/correlative-in-ope rando-studies-of-abusive-cycling-conditions-for-liion-batteries/A240CFD43A6425327435AC1E8 C15E650.

22. R. J. Copley, D. Cumming, Y. Wu, and R. S. Dwyer-Joyce, Journal of Energy Storage, 36, 102406 (2021) https://www.sciencedirect.com/science/article/pii/S2352152X21001602.

23. H. F. Pollard, Sound waves in solids, Pion, (1977).

24. G. E. Backus, J. Geophys. Res., 67, 4427-4440 (1962)

https://agupubs.onlinelibrary.wiley.com/doi/abs/10.1029/JZ067i011p04427.

25. M. C. Bhardwaj, Adv. Ceram. Mater., 1, 311-324 (1986).

26. M. Doyle, T. F. Fuller, and J. Newman, J. Electrochem. Soc. (1993)

http://jes.ecsdl.org/content/140/6/1526.short.

27. M. Doyle, J. Newman, A. S. Gozdz, and C. N. Schmutz, Journal of the (1996)

http://jes.ecsdl.org/content/143/6/1890.short.

28. T. F. Fuller, M. Doyle, and J. Newman, J. Electrochem. Soc. (1994)

http://jes.ecsdl.org/content/141/1/1.short.

29. Y. Qi, H. Guo, L. G. Hector, and A. Timmons, J. Electrochem. Soc., 157, A558-A566 (2010) http://jes.ecsdl.org/content/157/5/A558.abstract.

30. J. Xu, B. Liu, and D. Hu, Sci. Rep., 6, 21829 (2016) http://dx.doi.org/10.1038/srep21829.

31. Y. Qi, L. G. Hector, C. James, and K. J. Kim, J. Electrochem. Soc., 161, F3010-F3018 (2014) http://jes.ecsdl.org/content/161/11/F3010.abstract.

32. J. G. Swallow, W. H. Woodford, F. P. McGrogan, N. Ferralis, Y. M. Chiang, and K. J. Van Vliet, J. Electrochem. Soc., 161, F3084-F3090 (2014) 
http://jes.ecsdl.org/content/161/11/F3084.abstract.

33. J. Christensen and J. Newman, J. Solid State Electrochem. (2006)

https://link.springer.com/article/10.1007/s10008-006-0095-1.

34. J. Newman and W. Tiedemann, AIChE J. (1975)

https://aiche.onlinelibrary.wiley.com/doi/abs/10.1002/aic.690210103.

35. W. Ai, L. Kraft, J. Sturm, A. Jossen, and B. Wu, J. Electrochem. Soc., 167 (2020)

http://jes.ecsdl.org/content/167/1/013512.abstract.

36. L. Kraft, J. B. Habedank, A. Frank, A. Rheinfeld, and A. Jossen, J. Electrochem. Soc., 167 (2020) http://jes.ecsdl.org/content/167/1/013506.abstract.

37. M. Maiza, Y. Mammeri, D. A. Nguyen, N. Legrand, P. Desprez, A. A. and Franco, J. Power Sources, 423, 263-270 (2019) http://www.sciencedirect.com/science/article/pii/S0378775319302356.

38. D. A. Steingart, S. Bhadra, A. G. Hsieh, B. Hertzberg, P. J. Gjeltema, C. W. Rowley, US Patent (2016)

https://patentimages.storage.googleapis.com/47/51/77/5931e9771dac09/US20160223498A1.pd f.

39. A. G. Hsieh, S. Bhadra, B. J. Hertzberg, P. J. Gjeltema, A. Goy, J. W. Fleischer, and D. A. Steingart, Energy Environ. Sci., 8, 1569-1577 (2015)

https://pubs.rsc.org/en/content/articlehtml/2015/ee/c5ee00111k.

40. S. Bhadra, A. G. Hsieh, M. J. Wang, B. J. Hertzberg, and D. A. Steingart, J. Electrochem. Soc., 163, A1050-A1056 (2016) http://jes.ecsdl.org/content/163/6/A1050.abstract.

41. S. Bhadra, B. J. Hertzberg, A. G. Hsieh, M. Croft, J. W. Gallaway, B. J. Van Tassell, M. Chamoun, C. Erdonmez, Z. Zhong, T. Sholklapper, and D. A. Steingart, J. Mater. Chem. A Mater. Energy Sustain., 3, 9395-9400 (2015)

https://pubs.rsc.org/en/content/articlehtml/2015/ta/c5ta01576f.

42. P. Ladpli, C. Liu, F. Kopsaftopoulos, and F. Chang, in 2018 IEEE Transportation Electrification Conference and Expo, Asia-Pacific (ITEC Asia-Pacific),, p. 1-5 (2018) http://dx.doi.org/10.1109/ITEC-AP.2018.8433297.

43. J. B. Robinson, M. Maier, G. Alster, T. Compton, D. J. Brett, and P. R. Shearing, Phys. Chem. Chem. Phys., 21, 6354-6361 (2019) http://dx.doi.org/10.1039/c8cp07098a.

44. Y.-S. Chou, N.-Y. Hsu, K.-T. Jeng, K.-H. Chen, and S.-C. Yen, Appl. Energy, 182, 253-259 (2016) http://www.sciencedirect.com/science/article/pii/S0306261916312259.

45. C. A. Swoboda, D.R. Fredrickson, S.D. Gabelnick, P.H. Cannon, F. Hornstra, N.P. Yao, K.A. Phan, M.K. Singleterry, IEEE Transactions on Sonics and Ultrasonics, 30, 69-77 (1983) http://dx.doi.org/10.1109/T-SU.1983.31389.

46. M. Broussely, P. Biensan, F. Bonhomme, P. Blanchard, S. Herreyre, K. Nechev, and R. J. Staniewicz, J. Power Sources, 146, 90-96 (2005) 
http://www.sciencedirect.com/science/article/pii/S0378775305005082.

47. J. H. Lee, H. M. Lee, and S. Ahn, J. Power Sources, 119-121, 833-837 (2003)

http://www.sciencedirect.com/science/article/pii/S0378775303002817.

48. D. Liu, Y. Wang, Y. Xie, L. He, J. Chen, K. Wu, R. Xu, and Y. Gao, J. Power Sources, 232 , 29-33 (2013) http://www.sciencedirect.com/science/article/pii/S0378775313000335.

49. N. Nitta, F. Wu, J. T. Lee, and G. Yushin, Mater. Today, 18, 252-264 (2015)

http://www.sciencedirect.com/science/article/pii/S1369702114004118.

50. Y. Ein-Eli, B. Markovsky, D. Aurbach, Y. Carmeli, H. Yamin, and S. Luski, Electrochim. Acta, 39, 2559-2569 (1994) http://www.sciencedirect.com/science/article/pii/0013468694002215.

51. R. Deshpande, M. Verbrugge, Y.-T. Cheng, J. Wang, and P. Liu, J. Electrochem. Soc., 159, A1730-A1738 (2012) http://jes.ecsdl.org/content/159/10/A1730.abstract.

52. R. A. Leising, M. J. Palazzo, E. S. Takeuchi, and K. J. Takeuchi, J. Power Sources, 97-98, 681-683 (2001) http://www.sciencedirect.com/science/article/pii/S0378775301005985.

53. S. Koch, A. Fill, and K. P. Birke, J. Power Sources, 398, 106-112 (2018)

http://www.sciencedirect.com/science/article/pii/S0378775318307687.

54. S. K. Kumar, A. A. B. M. Abduh, O. Sabih, and R. Yazami, J. Electrochem. Soc., 165, A674-A679 (2018) http://jes.ecsdl.org/content/165/3/A674.abstract.

55. X. Tang, Y. Wang, K. Yao, Z. He, and F. Gao, J. Power Sources, 440, 227141 (2019) http://www.sciencedirect.com/science/article/pii/S0378775319311346.

56. J. Cannarella, X. Liu, C. Z. Leng, P. D. Sinko, G. Y. Gor, and C. B. Arnold, J. Electrochem. Soc., 161, F3117-F3122 (2014) http://jes.ecsdl.org/content/161/11/F3117.abstract.

57. Z. J. Schiffer, J. Cannarella, and C. B. Arnold, J. Electrochem. Soc., 163, A427-A433 (2016) http://jes.ecsdl.org/content/163/3/A427.abstract.

58. J. Cannarella and C. B. Arnold, J. Power Sources, 269, 7-14 (2014)

http://www.sciencedirect.com/science/article/pii/S0378775314010453.

59. J. Cannarella and C. B. Arnold, J. Power Sources, 245, 745-751 (2014)

http://www.sciencedirect.com/science/article/pii/S037877531301197X.

60. X. M. Liu and C. B. Arnold, J. Electrochem. Soc., 163, A2501-A2507 (2016)

http://jes.ecsdl.org/content/163/13/A2501.abstract.

61. N. P. Wagner, K. Asheim, F. Vullum-Bruer, and A. M. Svensson, J. Power Sources, 437, 226884 (2019) http://www.sciencedirect.com/science/article/pii/S0378775319308778.

62. W. Diao, S. Saxena, and M. Pecht, J. Power Sources, 435, 226830 (2019)

http://www.sciencedirect.com/science/article/pii/S0378775319308237.

63. N. A. Samad, Y. Kim, J. B. Siegel, and A. G. Stefanopoulou, J. Electrochem. Soc., 163, 
A1584-A1594 (2016) http://jes.ecsdl.org/content/163/8/A1584.abstract.

64. S. Mohan, Y. Kim, J. B. Siegel, N. A. Samad, and A. G. Stefanopoulou, J. Electrochem. Soc., 161, A2222-A2231 (2014) http://jes.ecsdl.org/content/161/14/A2222.abstract.

65. A. Raj, C. C. Dickerson, S. C. Nagpure, and S. Kim, Journal of The (2020) https://iopscience.iop.org/article/10.1149/1945-7111/ab6439/meta. 


\section{LIST OF FIGURES}
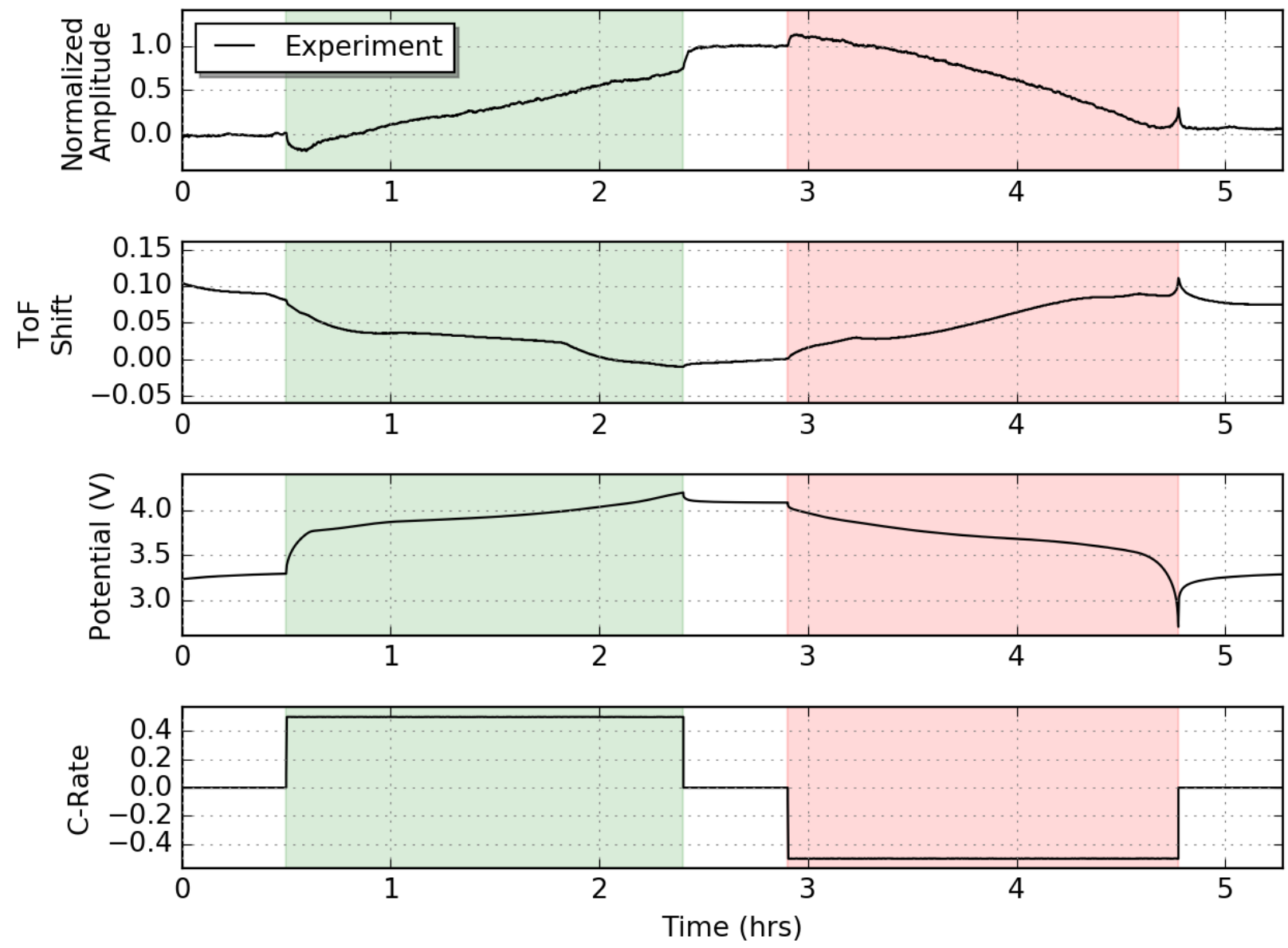

Figure 1. An example of ultrasonic acoustic data collected in conjunction with electrochemical data from the battery cycles, with a green background corresponding to cell charging and a red background corresponding to cell discharging (format maintained for latter figures). The top two panels contain ultrasonic acoustic data, the normalized amplitude and the Time of Flight (ToF) Shift, while the bottom two panels contain electrochemical data, the Potential and C-Rate based on nominal capacity. The acoustic amplitude is normalized such that a fully discharged state at rest corresponds to a value of 0.0 , while a fully charged state at rest corresponds to a value of 1.0. Note that upon application of current, the normalized amplitude values briefly exceed the bounds of 0.0 and 1.0, trends that are not seen in the ToF Shift or electrochemical data. 


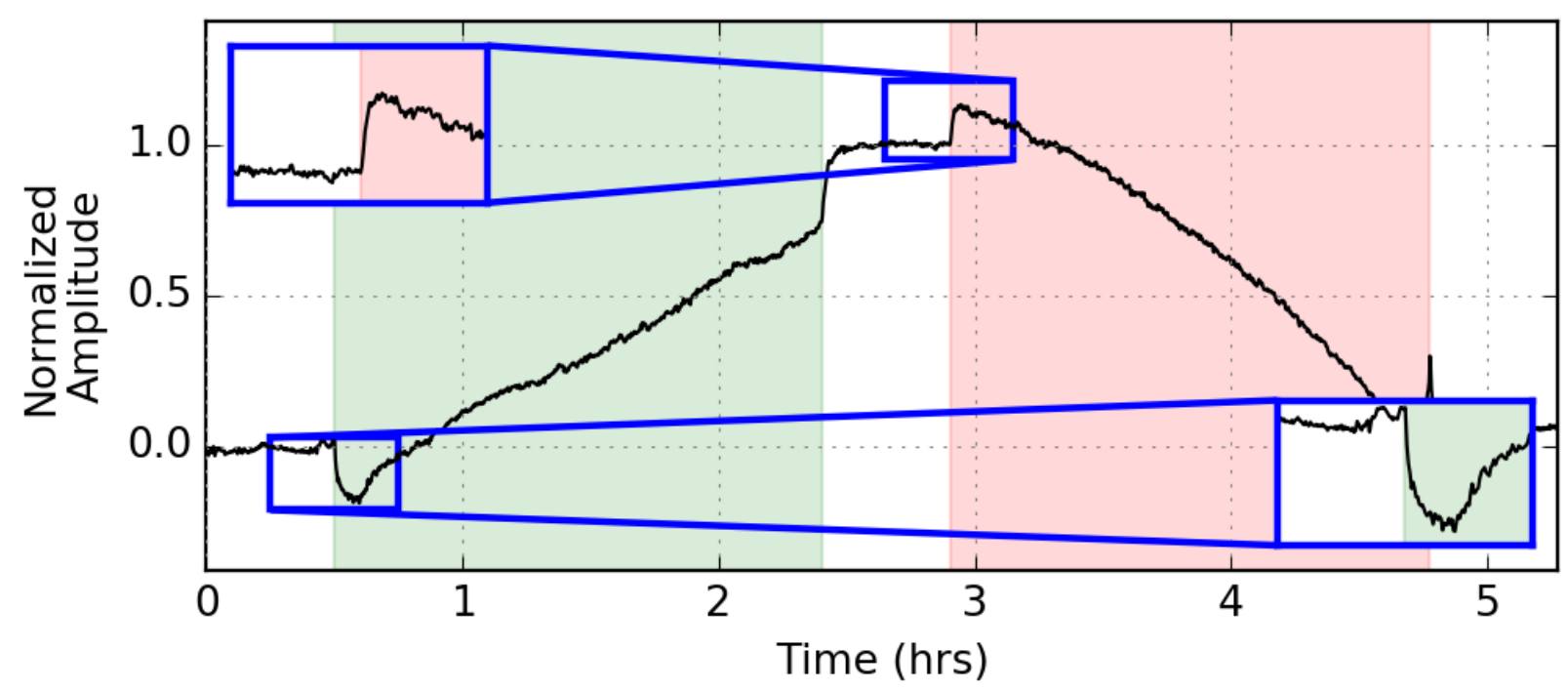

Figure 2. The initial jump in normalized amplitude is further shown in the zoomed inlets in the upper left and lower right corners. These rapid changes occur over roughly one to two minutes before the trend reverses course to a steady, linear trend. When current is no longer applied, the normalized amplitude jumps back in the opposite direction of its initial jump, suggesting a bulk shifting of the amplitude during charging and discharging. 


\section{Charging}

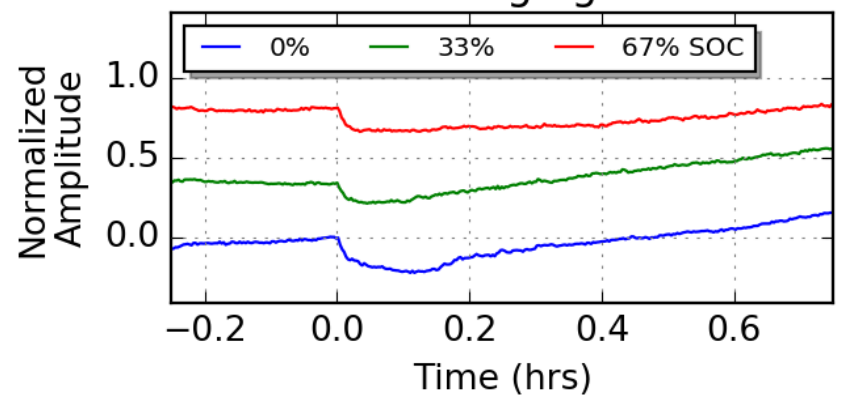

Discharging

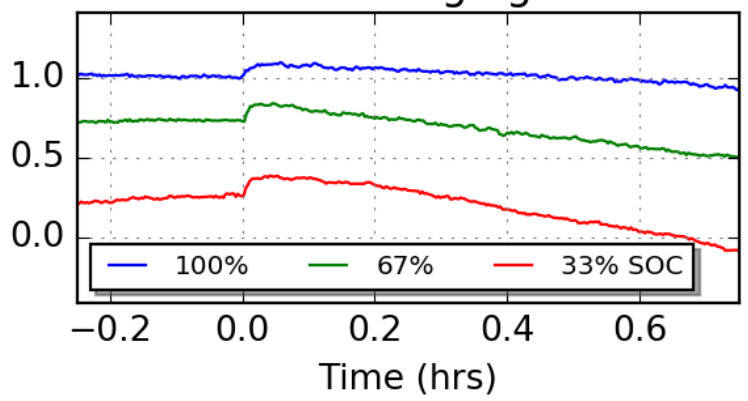

Figure 3. The bulk shift in normalized amplitude is shown to be independent of initial State of Charge (SoC). In the panel on the left, three initial charging conditions were chosen $(0 \%, 33 \%$, and $67 \%$ SoC) while the panel on the right shows three initial discharging conditions $(100 \%$, $67 \%$, and $33 \%$ SoC). These data were collected from the same battery undergoing a series of one hour $\mathrm{C} / 3$ discharges along with 30 minute rest periods in between, in order to properly normalize the amplitude across all experiments. The bulk shift changes little with SoC, suggesting this effect is the result of an SoC-invariant material (such as the electrolyte). 


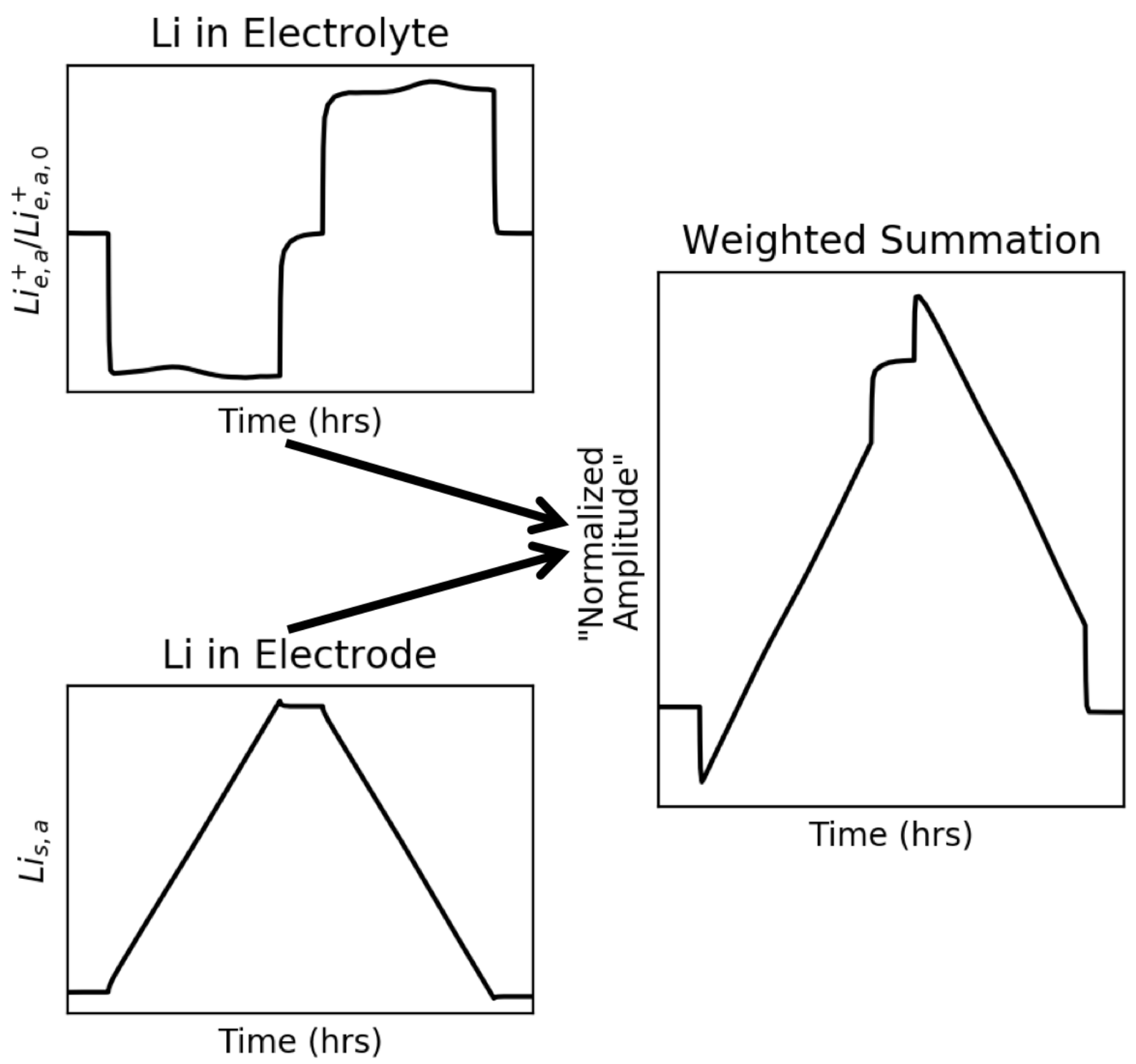

Figure 4. Dualfoil model simulation data show similar trends to what is seen in the normalized amplitude. In the upper left panel, changes to lithium ion concentration in the electrolyte around anode particles in the Dualfoil model are shown (normalized by an initial value of 1000 $\mathrm{mol} / \mathrm{m}^{\wedge} 3$ ). In the lower left panel, changes to the stoichiometry number $x$ in $\mathrm{Li}_{x} \mathrm{C}_{6}$ are shown (a proxy for changes in solid-state concentration of lithium in the anode particles). These values are combined via a weighted summation to produce the panel on the right, showing very similar trends to experimental normalized amplitude and thus being dubbed the "Normalized Amplitude" for the Dualfoil model. 

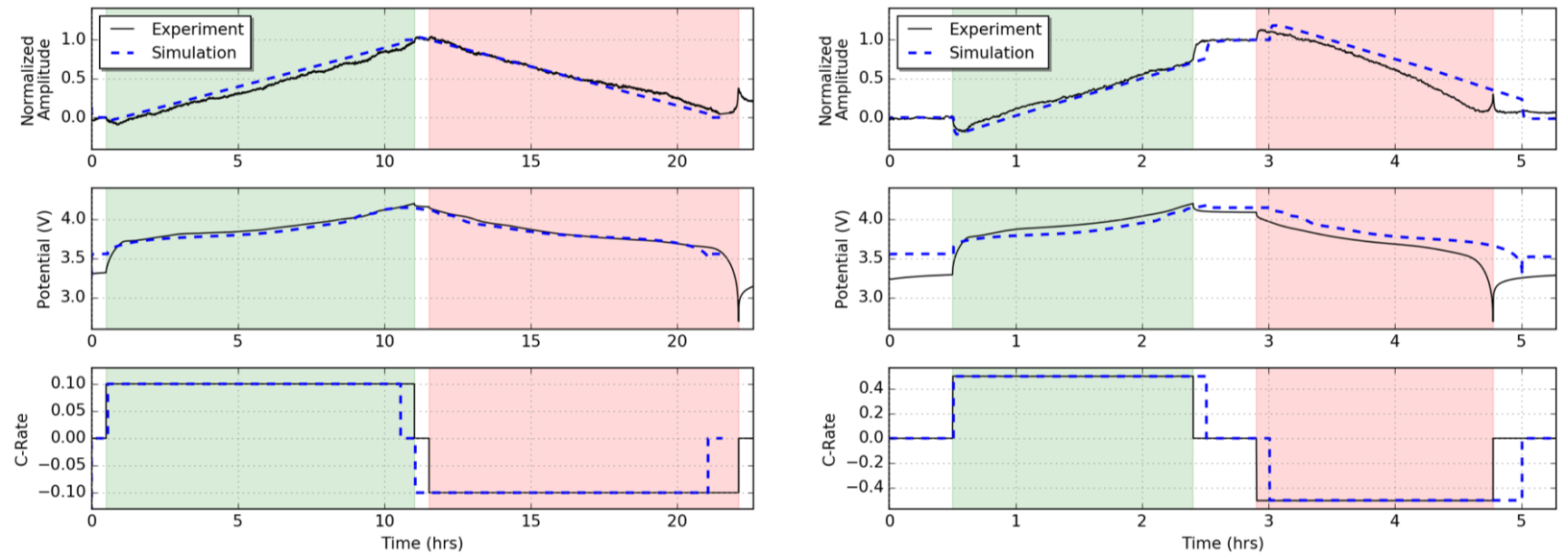

Figure 5. The "Normalized Amplitude" of Fig. 4 is compared with $\mathrm{C} / 10$ rate (left panel) and C/2 rate (right panel) experimental data, showing much agreement at both rates. Note the slight over- and under-performance of the experimental data for the $\mathrm{C} / 10$ and $\mathrm{C} / 2$ rates, respectively. This explains the shifting of the simulation data with respect to the experimental data after the initial charging step for both cases. 

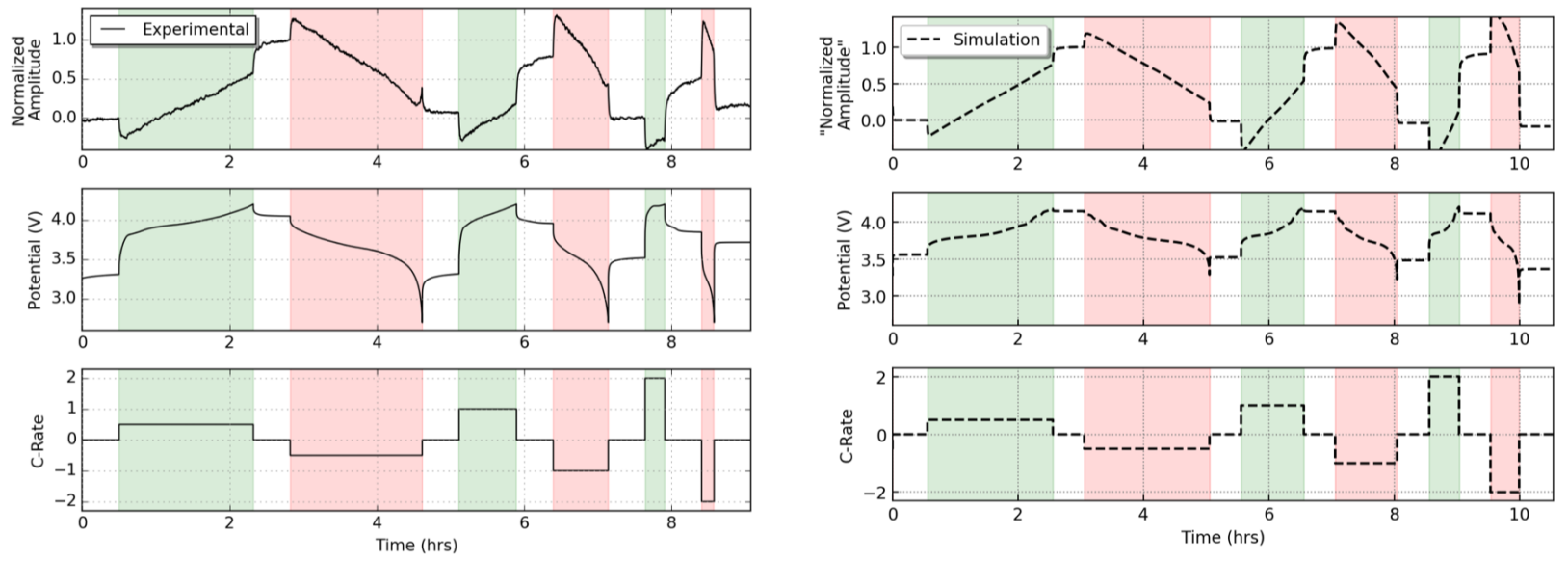

Figure 6. Dualfoil model simulation data is further compared to experimental data at higher rates. The panel on the left shows experimental data for a successive $C / 2,1 \mathrm{C}$, and $2 \mathrm{C}$ rate charge and discharge. The panel on the right shows Dualfoil model simulation data for the same cycling protocol, showing similar trends for $\mathrm{C} / 2$ and $1 \mathrm{C}$ data. The $2 \mathrm{C}$ data trends are different for charging; however, these particular LiB pouch cells were only rated for a $1 \mathrm{C}$ charge. Thus, other reactions may explain the discrepancy there. 


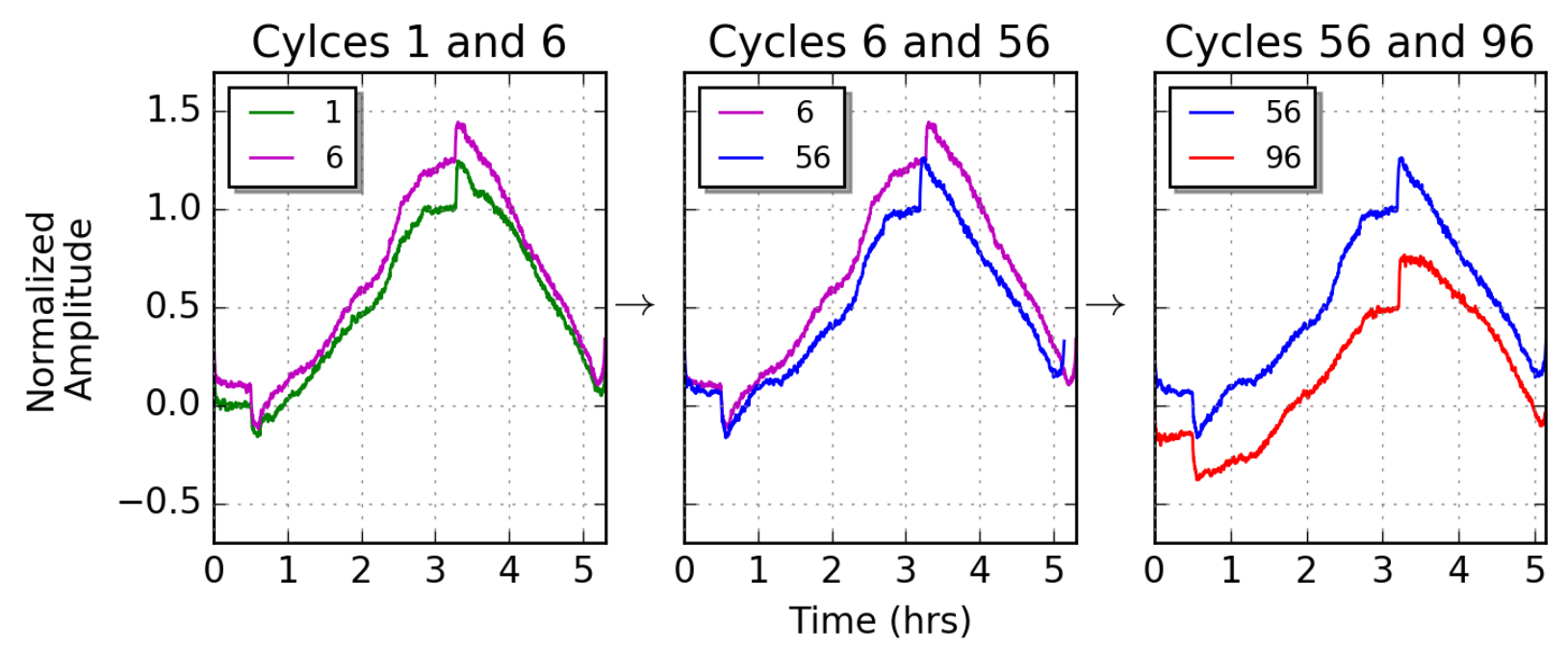

Figure 7. Normalized amplitude progression over many cycles is shown. The left panel shows normalized amplitude for early cycles 1 and 6 , the middle panel shows normalized amplitude after 56, while the right panel shows normalized amplitude after 96 cycles. In the first few cycles, the normalized amplitude at every point (including resting) is shown to increase. However, this increase levels out around cycle 6; afterwards, the normalized amplitude begins a steady decrease at every point (while still maintaining a roughly consistent value at bottom of charge resting). Around cycle 80 , this trend breaks down and a larger shift down in normalized amplitude is seen at all points, particularly at bottom of charge resting. The data is consistent with gassing occurring around cycle 80 . The result of this gassing is not only the drop in normalized amplitude at cycle 96, but also changes to the bulk shifting of the normalized amplitude. As opposed to the bulk shift occurring over one to two minutes, the bulk shift occurs over almost 30 minutes for cycle 96. 


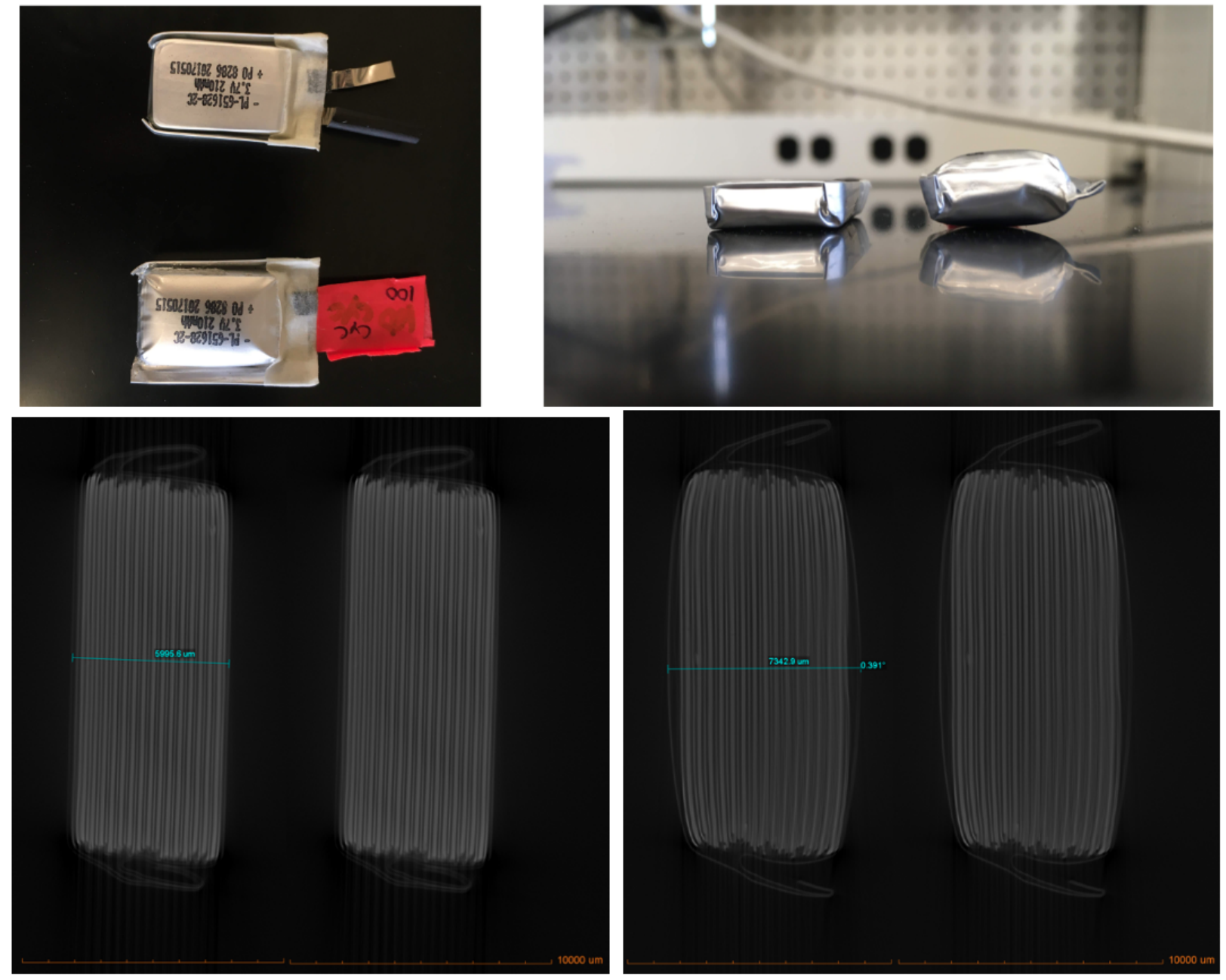

Figure 8. Comparison of a fresh cell (top in the top left image, left in the top right image) to the cell from Fig. 7 (bottom in the top left image, right in the top right image). Note that the cycled cell is quite bloated, typical of gas formation. TXM data of these cells are shown in the bottom images, with the fresh cell on the bottom left and the gassed cell on the bottom right. Scale bars of $10000 \mu \mathrm{m}$ are provided at the bottom of these images. Cell thickness measurements were made for the fresh cell $(5995.6 \mu \mathrm{m})$ and the bloated cell $(7342.9 \mu \mathrm{m})$. 

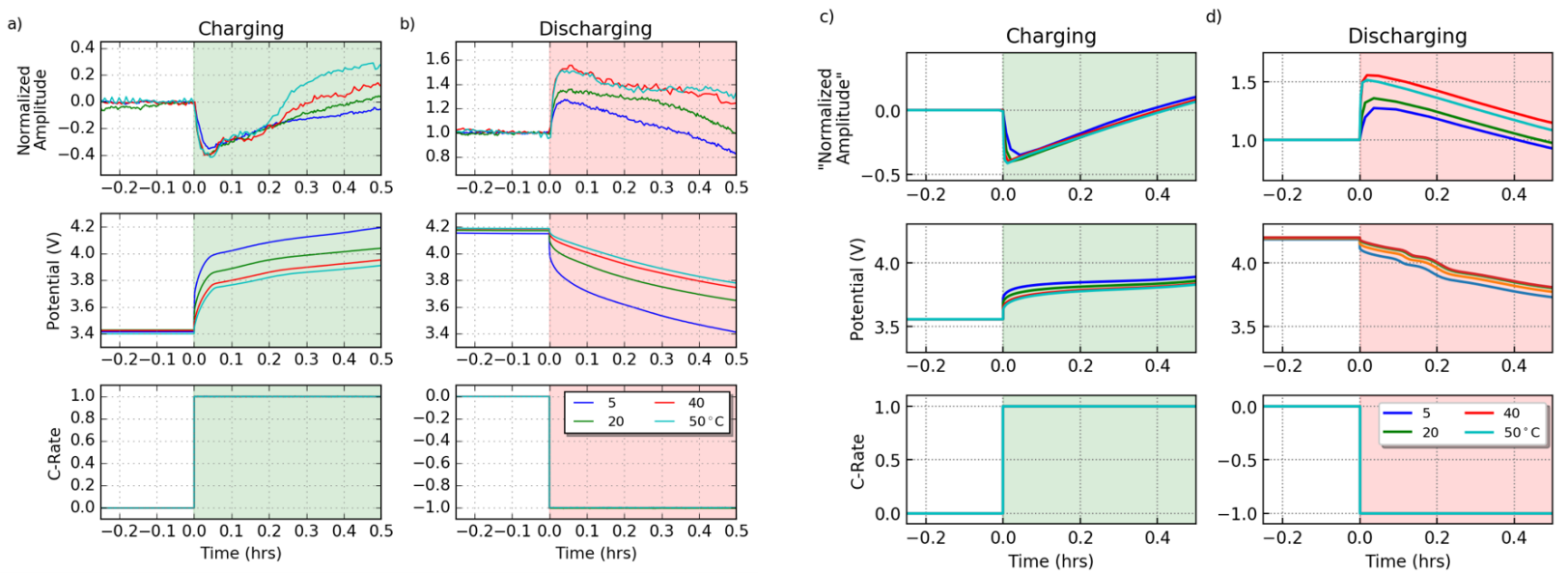

Figure 9. Experimental data $(a, b)$ and Dualfoil model simulation $(c, d)$ data for a $1 \mathrm{C}$ charge $(a$, c) and discharge (b, d) at various temperatures $\left(5,20,40\right.$, and $\left.50^{\circ} \mathrm{C}\right)$. In order to best fit experimental and simulated data, the value of $\xi$ was changed. The initial value of $\xi=2.82$ is no longer applicable in every case, and therefore $\xi$ is changed in the Dualfoil model to match peaks seen in experiments. While the Dualfoil model captures the temperature behavior with varying $\xi$, discrepancies lie after the initial bulk shifting. 


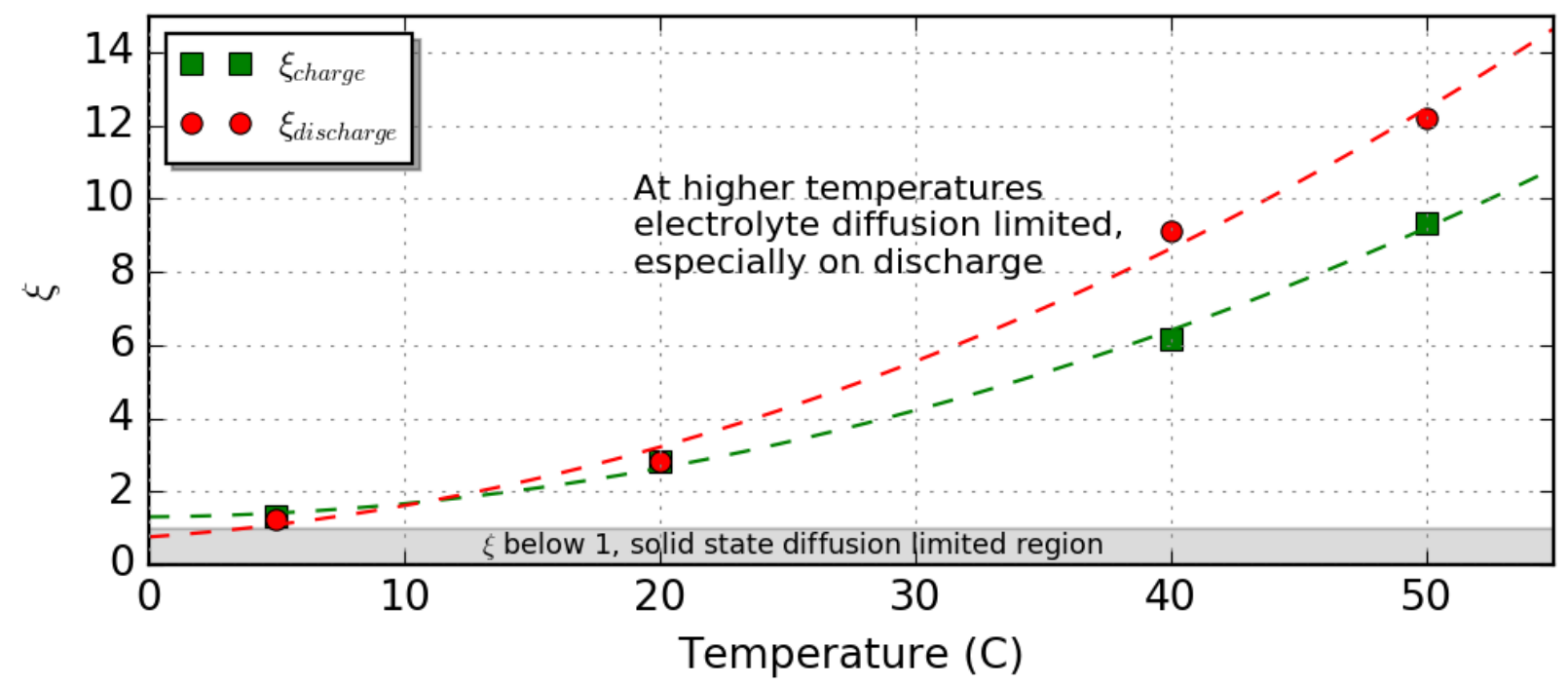

Figure 10. $\xi$ data from Fig. 9 at charge (green) and discharge (red) that was used to match simulated data with experimental data. Note that $\xi$ is always greater than one, suggesting that changes in the electrolyte are dominating the bulk shifting trend, particularly at elevated temperatures. Trend lines for $\xi$ in the charged (dotted green) and discharged (dotted red) states are shown. In order to fit both charge and discharge experimental data, it is noted that $\xi$ diverges to different values for charge and discharge at elevated temperatures. 


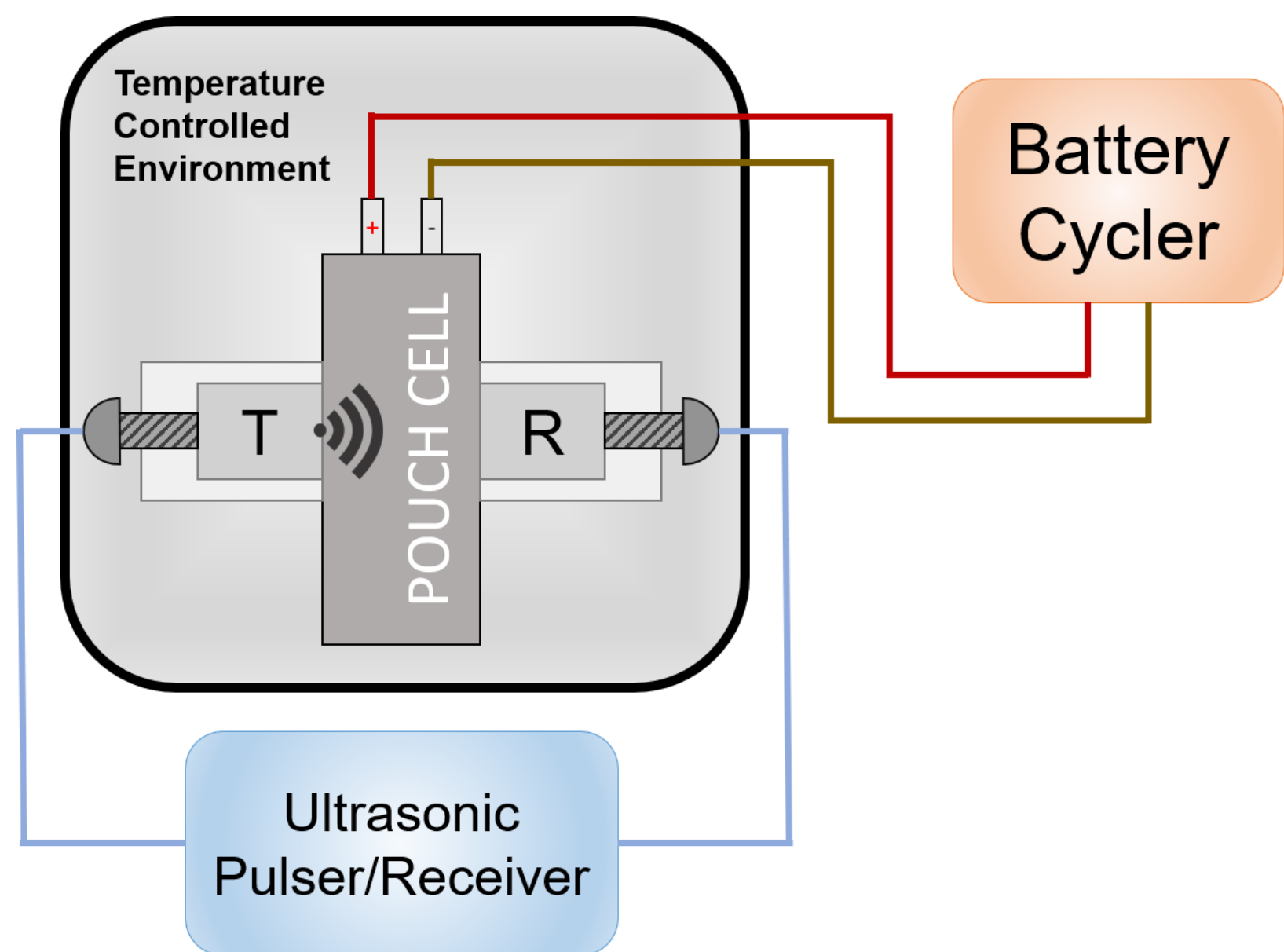

Figure S1. A schematic of the battery cycler and ultrasonic pulser-receiver setup. The ultrasonic pulser records data prior to, during, and post cycling of the commercial lithium-ion battery. The battery and ultrasonic transducers are placed in a temperature controlled environment to ensure consistent testing conditions. 

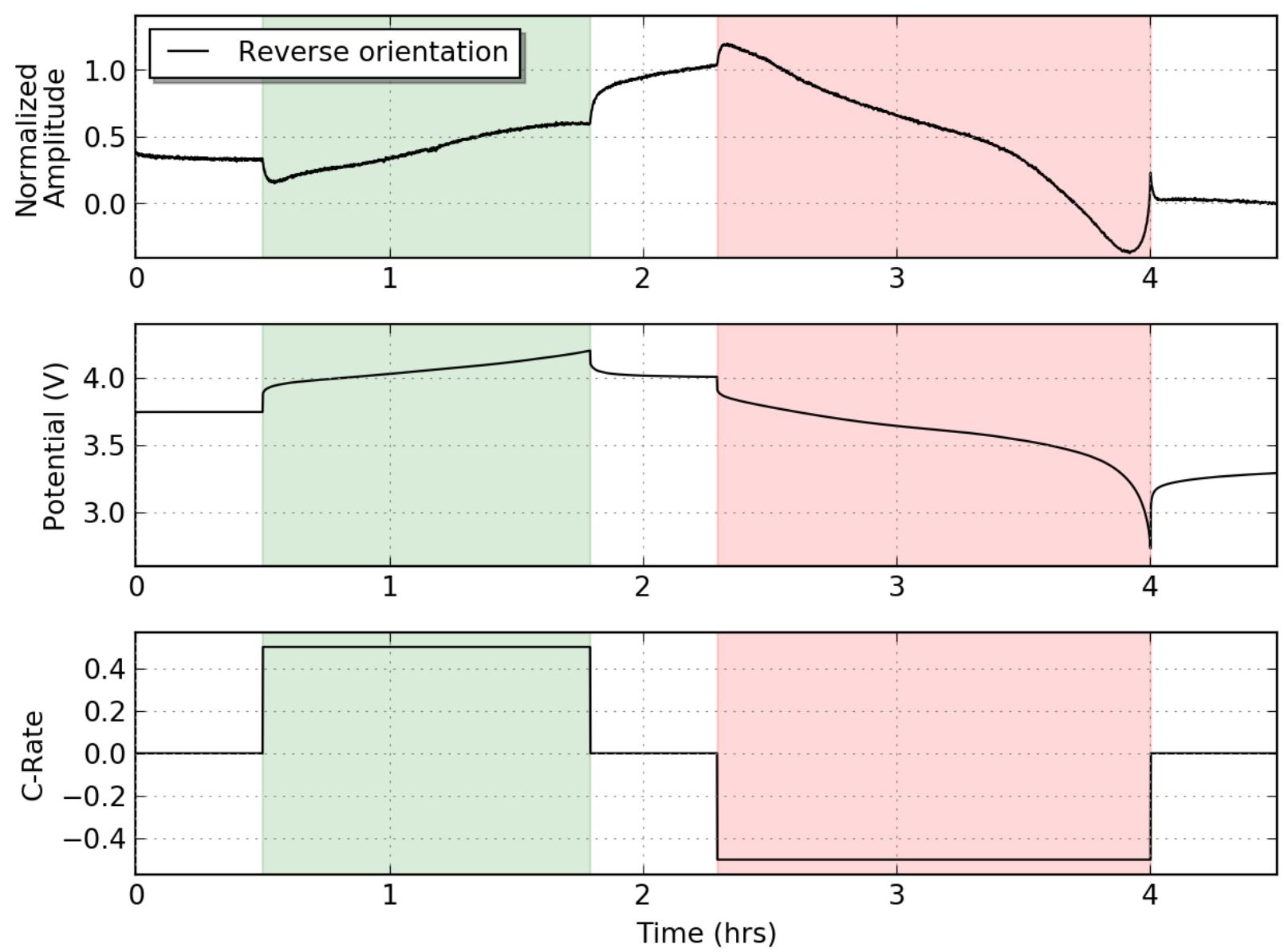

Figure S2. Normalized amplitude trends when the ultrasonic acoustic transducers are reversed in orientation. Note that the cell starts at roughly $40 \%$ SoC for the initial charge. 

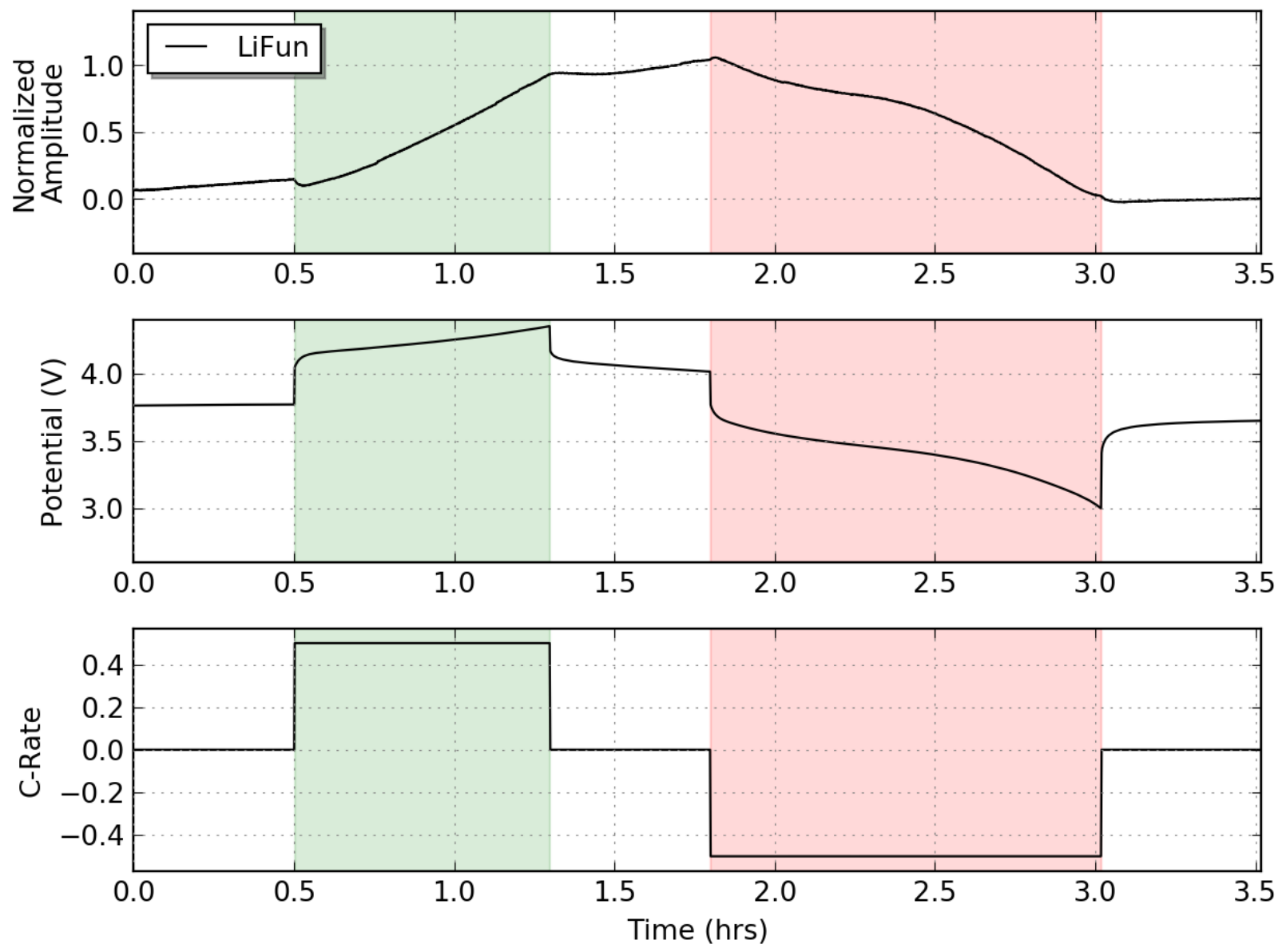

Figure S3. Normalized amplitude trends when the ultrasonic acoustic transducers are from a different manufacturer (LiFun) and the data comes from a different ultrasonic pulser setup. Note that the cell starts at roughly $50 \%$ SoC for the initial charge. 

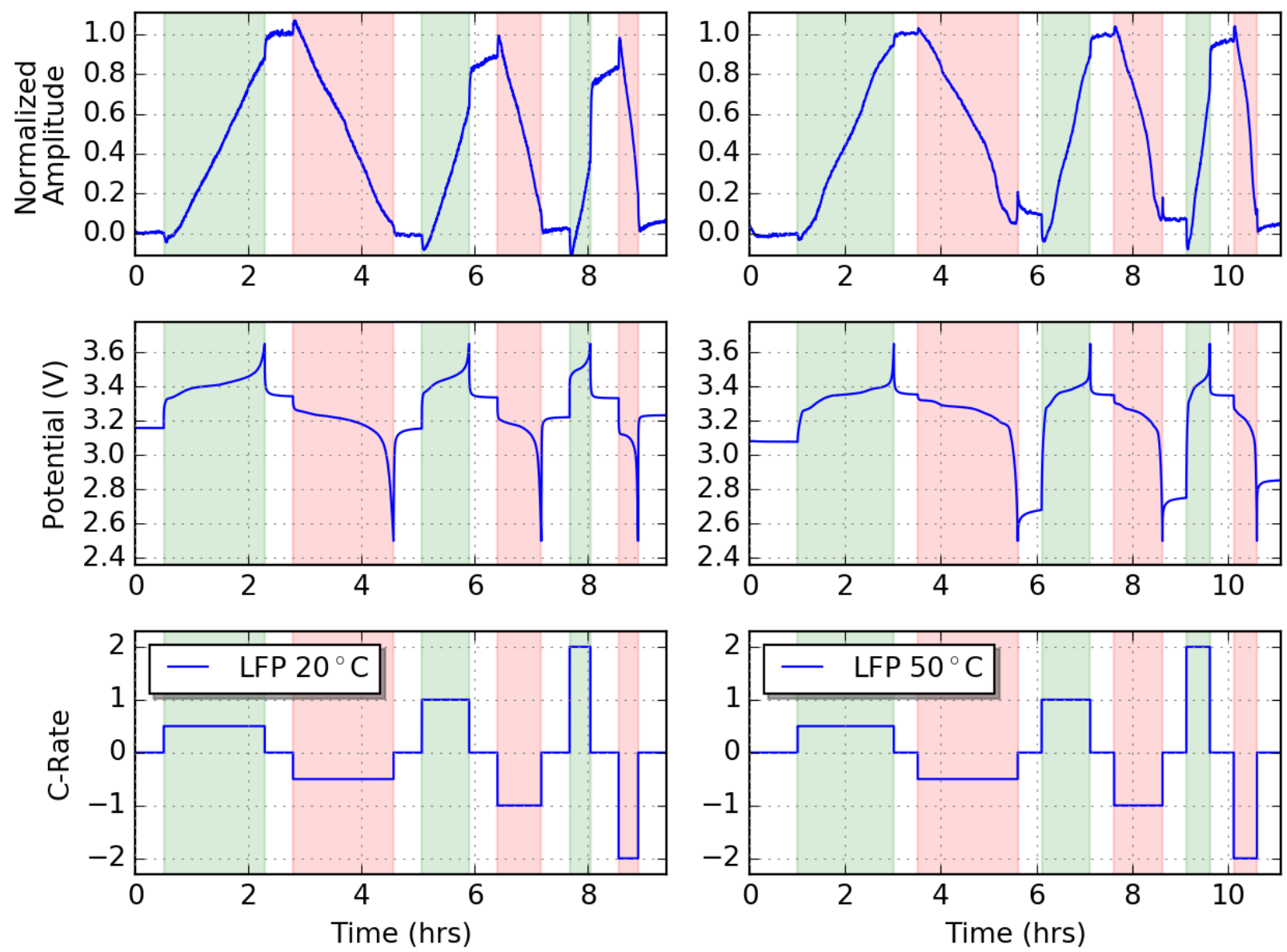

Figure S4. Normalized Amplitude trends for cells of a different cathode chemistry (lithium iron phosphate, LFP) and same anode chemistry (graphite) at $20^{\circ} \mathrm{C}$ (left panels) and $50^{\circ} \mathrm{C}$ (right panels). Each cell undergoes successive $\mathrm{C} / 2,1 \mathrm{C}$, and $2 \mathrm{C}$ cycling protocol. The normalized amplitude trends, including the bulk shifting, are similar to those seen in C/LCO cells, suggesting these trends are the result of the graphite anode. 

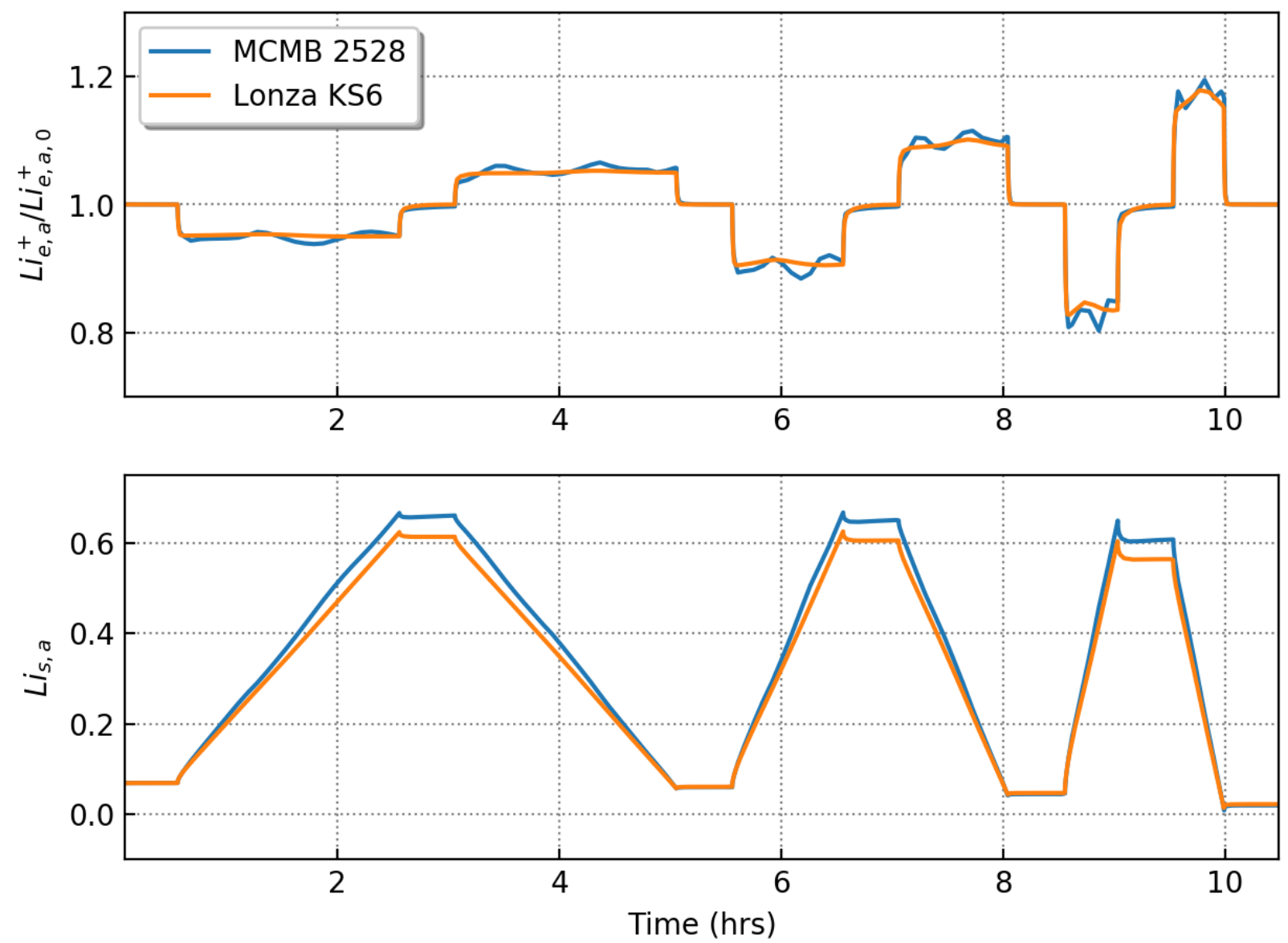

Figure S5. Comparison of Dualfoil model simulation data for the default negative electrode material, MCMB 2528 Bellcore graphite, and Lonza KS6 Bellcore graphite. The top panel shows changes to the lithium-ion concentration in the electrolyte around the anode particles (normalized by its initial value). The bottom panel shows changes to the stoichiometry number $x$ in $\mathrm{Li}_{\mathrm{x}} \mathrm{C}_{6}$. The simulation protocol is successive $\mathrm{C} / 2,1 \mathrm{C}$, and $2 \mathrm{C}$ cycles. The MCMB 2528 Bellcore graphite shows oscillations in the lithium ion concentration in the electrolyte around the anode particles, particularly at higher rates, and this is the result of over-fitting of lithium staging in the graphite electrode. Lonza KS6 Bellcore graphite is chosen in our Dualfoil model due to the suppressed changes as a result of lithium staging. 